\title{
Altered gut-liver axis and hepatic adiponectin expression in OSAS: novel mediators of liver injury in paediatric non-alcoholic fatty liver
}

\author{
Valerio Nobili, ${ }^{1}$ Anna Alisi, ${ }^{2}$ Renato Cutrera, ${ }^{3}$ Guido Carpino, ${ }^{4}$ Cristiano De Stefanis, ${ }^{2}$ \\ Valentina D'Oria, ${ }^{5}$ Rita De Vito, ${ }^{6}$ Salvatore Cucchiara, ${ }^{7}$ Eugenio Gaudio, ${ }^{8}$ \\ Giovanni Musso ${ }^{9}$
}

- Additional material is published online only. To view please visit the journal online (http://dx.doi.org/10.1136/ thoraxjnl-2015-206782).

For numbered affiliations see end of article.

\section{Correspondence to}

Dr Giovanni Musso, Gradenigo Hospital C.so Regina Margherita 8, Turin 10132 , Italy; giovanni_musso@yahoo.it

Received 8 January 2015 Revised 8 May 2015 Accepted 26 May 2015 Published Online First 11 June 2015

\section{SLinked}

- http://dx.doi.org/10.1136/ thoraxjnl-2015-207212

CrossMark

To cite: Nobili V, Alisi $A$ Cutrera $\mathrm{R}$, et al. Thorax 2015;70:769-781.

\section{ABSTRACT}

Background Mechanism(s) connecting obstructive sleep apnoea syndrome (OSAS) to liver injury in paediatric non-alcoholic fatty liver disease (NAFLD) are unknown. We hypothesised alterations in gut-liver axis and in the pool and phenotype of hepatic progenitor cells (HPCs) may be involved in OSAS-associated liver injury in NAFLD.

Methods Eighty biopsy-proven NAFLD children (age, mean $\pm S D, 11.4 \pm 2.0$ years, $56 \%$ males, body mass index $z$-score $1.95 \pm 0.57$ ) underwent a clinicalbiochemical assessment, with measurement of insulin sensitivity, plasma cytokines, lipopolysaccharide (LPS), an intestinal permeability test and a standard polysomnography. Hepatic toll-like receptor (TLR)-4 expression by liver-resident cells and overall number and expression of resistin and adiponectin by HPCs were assessed by immunofluorescence and immunohistochemistry. OSAS was defined by an apnoea/ hypopnoea index $\geq 1$.

Results OSAS was characterised by an increased intestinal permeability and endotoxemia, coupled with TLR-4 upregulation in hepatocytes, Kupffer and hepatic stellate cells (HSCs) and by an expansion of an adiponectin-deficient HPC pool, key features of steatohepatitis and fibrosis.

The duration of haemoglobin desaturation $\left(\mathrm{SaO}_{2}\right.$ $<90 \%)$ independently predicted intestinal permeability ( $\beta$ : 0.396; $p=0.026)$, plasma LPS ( $\beta$ : 0.358; $p=0.008$ ) and TLR-4 expression by hepatocytes ( $\beta$ : 0.332 ; $p=0.009)$, Kupffer cells $(\beta: 0.357 ; p=0.006)$ and HSCs ( $\beta: 0.445 ; p=0.002$ ).

$\mathrm{SaO}_{2}<90 \%$ predicted also HPC number ( $\beta$ : 0.471 ; $\mathrm{p}=0.001)$ and impaired adiponectin expression by HPC pool ( $\beta$ : $-0.532 ; p=0.0009$ ).

These relationships were observed in obese and nonobese children.

Conclusions In paediatric NAFLD, OSAS is associated with increased endotoxemia coupled with impaired gut barrier function, with increased TLR-4-mediated hepatic susceptibility to endotoxemia and with an expansion of an adiponectin-deficient HPC pool. These alterations may represent a novel pathogenic link and a potential therapeutic target for OSAS-associated liver injury in NAFLD.

\section{INTRODUCTION}

Non-alcoholic fatty liver disease (NAFLD) affects $10 \%$ of the general paediatric population and

\section{Key messages}

What is the key question?

- Are alterations in gut-liver axis involved in the pathogenesis of obstructive sleep apnoea syndrome (OSAS)-associated liver injury in paediatric non-alcoholic fatty liver disease (NAFLD)?

\section{What is the bottom line?}

- Recent data linked the presence and severity of OSAS to the presence and severity of NAFLD, but mechanism(s) connecting OSAS to liver injury are unclear.

\section{Why read on?}

- OSAS may promote liver injury on one side by impairing intestinal barrier function and promoting endotoxemia and on the other side by sensitising the liver to endotoxin and proinflammatory stimuli: these pathways may mediate OSAS-associated liver injury in NAFLD.

$50-70 \%$ of obese children, and its prevalence is rising along with the obesity epidemic. ${ }^{1}$ NAFLD encompasses a histological spectrum ranging from simple steatosis to non-alcoholic steatohepatitis (NASH): while the former has a benign hepatological course, NASH can progress to cirrhosis and hepatocellular carcinoma (HCC). ${ }^{2}$ Although NAFLD is generally a slowly progressive disease, approximately $8 \%$ of children undergoing liver biopsy for suspected NAFLD have cirrhosis, ${ }^{3}$ and onset of HCC on background NAFLD has been reported as early as age $7 .^{4}$ These data indicate the need for early recognition and treatment of NASH to prevent liver-related complications in adulthood, and recent American Gastroenterological Association/American College of Gastroenterology/ American Association for the Study of Liver Diseases guidelines state that onset of NAFLD in childhood may be at greater risk for severe liverrelated complications later in life. ${ }^{5}$

Obstructive sleep apnoea syndrome (OSAS) is increasingly recognised in children, affecting $8 \%$ of paediatric population and up to $78 \%$ of obese children. ${ }^{6}$ 
Besides being an emerging cardiometabolic risk factor, OSAS has been recently connected to the presence and severity of liver disease in paediatric NAFLD, independently of whole-body/ abdominal obesity, insulin resistance and metabolic syndrome (MS), ${ }^{8} 9$ but the mechanism(s) connecting OSAS to liver injury are unknown.

Current therapeutic approaches for paediatric OSAS have disappointing success rates, and elucidating mechanisms mediating hypoxia-associated liver injury would offer novel therapeutic opportunities for OSAS-associated NASH and fibrosis. ${ }^{10}$ Two pathways for liver disease progression have been recently identified in NAFLD.

The first is an altered gut-liver interaction, including an impaired intestinal barrier integrity with enhanced lipopolysaccharide (LPS) translocation and increased plasma endotoxemia, leading to chronic low-grade inflammation and liver injury through activation of the LPS-toll-like-receptor (TLR)-4 signalling pathway. ${ }^{11} 12$

The second pathway for liver disease progression is an expansion of hepatic progenitor stem cell (HPC) pool and an altered pattern of adipokine expression by HPCs and hepatocytes, which appear more tightly related to liver injury than circulating adipokines. $^{13}$ The mechanisms regulating gut-liver interaction and HPCs pool and phenotype in NAFLD are unknown.

Chronic intermittent hypoxia $(\mathrm{CIH})$ profoundly affects intestinal epithelial and entero-endocrine cell integrity and function experimentally, and hypoxia is a major regulator of progenitor cell proliferation and phenotype in diverse disease models. ${ }^{14-18}$

Currently, there are no in vitro or in vivo data on the role of gut-liver axis and of HPC compartment as a possible mediator of OSAS-associated liver injury in NAFLD.

We hypothesised that intermittent hypoxia affects liver injury by modulating gut-liver axis and/or HPC proliferation and adipokine phenotype in paediatric NAFLD.

\section{METHODS}

\section{Patients}

The study was performed at the Bambino Gesù Children's Hospital during the period January 2012 to January 2014.

Eighty-one consecutive children with an ultrasonographic diagnosis of NAFLD, persistently ( $\geq 6$ months) elevated serum aminotransferases and symptoms/signs suggestive of sleep apnoea, ${ }^{8}$ were prospectively seen and offered liver biopsy and polysomnography (PSG) as part of the experimental protocol, designed to evaluate the relationship of sleep disturbance with liver disease. ${ }^{18}$

Among these 81 patients, 80 children were assented and parents consented to participate. Hepatitis virus infection, other competing causes of hepatic steatosis and coeliac disease were excluded according to standard guidelines (see online supplementary file).

Body mass index (BMI), waist circumference and their SD score $\left(z\right.$-score) were calculated. ${ }^{19}{ }^{20}$ MS was defined as the presence of $\geq 3$ of the following five criteria: ${ }^{21}$ abdominal obesity as defined by a waist circumference $\geq 90$ th percentile for age and sex, ${ }^{20}$ hypertriglyceridemia as defined by triglycerides (TG) $>95$ th percentile for age and sex; ${ }^{22}$ low high-density lipoprotein cholesterol (HDL-C) as defined by $<5$ th percentile for age and sex; ${ }^{23}$ elevated blood pressure (BP) as defined by systolic or diastolic BP $>95$ th percentile for age and sex, ${ }^{24}$ and impaired fasting glucose, impaired glucose tolerance or type 2 diabetes mellitus. $^{25}$
Dietary record

Patients and responsible guardians underwent a $1 \mathrm{~h}$ nutritional interview with experienced dieticians, and their dietary intake was recorded as previously described. ${ }^{11} 13$ The patients received no treatment for NAFLD or OSAS before liver biopsy and polysomnography were performed.

\section{Laboratory assessment}

Aspartate aminotransferase, alanine transaminase, gammaglutamyl transpeptidase, total TG and total/HDL-C were assessed using standard laboratory methods. All participants underwent a standard oral glucose tolerance test, performed with $1.75 \mathrm{~g}$ of glucose $/ \mathrm{kg}$ of body weight (up to $75 \mathrm{~g}$ ). Two indices of insulin sensitivity were calculated as previously described: ${ }^{8}$ the homeostasis model assessment of insulin resistance and the insulin sensitivity index.

\section{Inflammatory markers and cytokines}

Serum C-reactive protein was determined via a high-sensitivity latex agglutination method on HITACHI 911 Analyser (Sentinel Ch., Milan).

Serum adiponectin, tumour necrosis factor- $\alpha$, interleukin- 6 , leptin, resistin (RayBiotech, Norcross, Georgia, USA) and retinol-binding protein-4 (Dade Behring, Newark, Delaware, USA) were measured by sandwich ELISA. Sensitivity and intra-assay and inter-assay CVs of each kit are detailed in online supplementary file).

\section{Markers of hepatocyte apoptosis and of extracellular matrix deposition}

Circulating cytokeratin (CK)18 fragments and hyaluronic acid, two validated markers of hepatocyte apoptosis and extracellular matrix deposition, respectively, in paediatric NAFLD ${ }^{6}$ were measured (see online supplementary file).

\section{Intestinal permeability test and plasma LPS}

Children underwent the previously described and validated intestinal permeability test with lactulose and mannitol, ${ }^{11}$ and the ratio of the fractional urine excretion of lactulose to the fractional urine excretion of mannitol (L/M ratio) was calculated. $\mathrm{L} / \mathrm{M}$ ratio, as an index of intestinal permeability, was entered as both a continuous and a dichotomous variable: an L/ $\mathrm{M}$ ratio of $\geq 0.03$ was considered abnormal based upon previously established references. 9

Plasma LPS concentration was measured by a commercially available kit (Amebocyte Lysate (LAL) LAL Chromogenic Endpoint Assay. Cambrex Limulus kit; Hycult Biotech, Uden, the Netherlands).

Liver histology: Liver biopsy was performed after an overnight fast, and specimens processed as detailed in online supplementary file. Biopsies were evaluated by a single blinded pathologist, with a long-time experience in the field. Steatosis, inflammation, hepatocyte ballooning and fibrosis were scored using the NAFLD Clinical Research Network criteria, as recently recommended (detailed in online supplementary file). ${ }^{5}$ Additionally, the presence of portal fibrosis, a distinctive feature of early fibrosis in paediatric NAFLD, ${ }^{1}$ was recorded.

Features of steatosis, lobular inflammation and hepatocyte ballooning were combined to obtain the NAFLD activity score (NAS). As recommended by current guidelines, biopsies were subdivided into not NASH and definite NASH subcategories, on the basis of overall injury pattern (steatosis, hepatocyte ballooning, inflammation), as well as the presence of additional 
Table 1 Clinical and biochemical features of study population, grouped according to the presence/absence of NASH ( $\mathrm{n}=80$ )

\begin{tabular}{|c|c|c|c|c|}
\hline Parameter & NAFLD $(n=80)$ & Not NASH $(n=31)$ & NASH $(n=49)$ & $\begin{array}{l}\text { P value } \\
\text { NASH versus not NASH }\end{array}$ \\
\hline Age (years) & $11.4 \pm 2.0$ & $11.7 \pm 1.8$ & $11.2 \pm 2.3$ & 0.251 \\
\hline Gender $\mathrm{n}(\%)$ males & $45(56 \%)$ & $20(65 \%)$ & $25(51 \%)$ & 0.237 \\
\hline BMI $\left(\mathrm{kg} / \mathrm{m}^{2}\right)$ & $27.7 \pm 5.1$ & $26.1 \pm 5.3$ & $28.4 \pm 4.8$ & 0.027 \\
\hline BMI z-score & $1.95 \pm 0.57$ & $1.72 \pm 0.59$ & $2.11 \pm 0.51$ & 0.003 \\
\hline Obese subjects $\mathrm{n}(\%)^{*}$ & $59(73 \%)$ & $18(58 \%)$ & $41(83 \%)$ & 0.017 \\
\hline Waist circumference (cm) & $89.4 \pm 4.6$ & $89.2 \pm 5.2$ & $89.6 \pm 4.4$ & 0.793 \\
\hline Waist circumference $z$-score & $1.99 \pm 0.78$ & $1.92 \pm 0.75$ & $2.04 \pm 0.73$ & 0.486 \\
\hline Systolic BP (mm Hg) & $112 \pm 12$ & $114 \pm 15$ & $111 \pm 12$ & 0.323 \\
\hline Diastolic BP (mm Hg) & $67 \pm 7$ & $68 \pm 9$ & $68 \pm 8$ & 0.793 \\
\hline Insulin $(\mu \mathrm{U} / \mathrm{mL})$ & $13.8 \pm 9.6$ & $15.7 \pm 10.4$ & $12.7 \pm 9.1$ & 0.172 \\
\hline Glucose (mg/dL) & $86 \pm 14$ & $86 \pm 12$ & $85 \pm 16$ & 0.815 \\
\hline HOMA-IR & $2.91 \pm 2.01$ & $3.20 \pm 2.04$ & $2.75 \pm 2.04$ & 0.274 \\
\hline ISI & $2.95 \pm 1.96$ & $3.49 \pm 2.43$ & $2.61 \pm 1.50$ & 0.213 \\
\hline Triglyceride (mg/dL) & $113 \pm 61$ & $100 \pm 49$ & $120 \pm 75$ & 0.199 \\
\hline Total cholesterol (mg/dL) & $160 \pm 34$ & $155 \pm 23$ & $163 \pm 36$ & 0.146 \\
\hline HDL-C (mg/dL) & $44 \pm 6$ & $43 \pm 8$ & $42 \pm 7$ & 0.616 \\
\hline Diabetes n (\%) & $2(2 \%)$ & $0 \%$ & $2(4 \%)$ & 0.512 \\
\hline Metabolic syndrome n (\%) & $13(16 \%)$ & $3(10 \%)$ & $10(20 \%)$ & 0.431 \\
\hline Abdominal obesity n (\%)† & $50(63 \%)$ & $20(65 \%)$ & $30(61 \%)$ & 0.814 \\
\hline Hypertension n (\%) & $11(14 \%)$ & $5(16 \%)$ & $6(12 \%)$ & 0.638 \\
\hline Hypertriglyceridemia n (\%) & $22(28 \%)$ & $6(19 \%)$ & $16(33 \%)$ & 0.298 \\
\hline Low HDL-C n (\%) & $22(27 \%)$ & $11(35 \%)$ & $11(22)$ & 0.542 \\
\hline IFG/diabetes n (\%) & $14(18 \%)$ & $5(16 \%)$ & $9(18 \%)$ & 0.874 \\
\hline Adiponectin (ng/mL) & $21.51 \pm 2.19$ & $22.89 \pm 2.23$ & $20.63 \pm 1.55$ & $<0.001$ \\
\hline Resistin (pg/mL) & $33755 \pm 37363$ & $29505 \pm 12257$ & $37233 \pm 29424$ & 0.170 \\
\hline $\mathrm{RBP}(\mathrm{mg} / \mathrm{mL})$ & $3.15 \pm 1.13$ & $3.07 \pm 1.11$ & $3.19 \pm 1.15$ & 0.646 \\
\hline C-reactive protein (mg/L) & $1.6 \pm 0.5$ & $1.5 \pm 0.4$ & $1.6 \pm 0.4$ & 0.617 \\
\hline IL-6 (pg/mL) & $11.3 \pm 5.7$ & $11.2 \pm 5.7$ & $11.3 \pm 5.9$ & 0.621 \\
\hline Leptin (ng/mL) & $19.3 \pm 8.9$ & $19.5 \pm 7.7$ & $19.1 \pm 9.7$ & 0.876 \\
\hline TNF- $\alpha(p g / m L)$ & $6.8 \pm 2.4$ & $6.8 \pm 2.1$ & $6.7 \pm 2.3$ & 0.834 \\
\hline L/M ratio & $0.04 \pm 0.04$ & $0.02 \pm 0.02$ & $0.06 \pm 0.04$ & $<0.001$ \\
\hline Pathological L/M ratio $\mathrm{n}(\%)$ & $28(35 \%)$ & $2(6 \%)$ & $26(53 \%)$ & $<0.001$ \\
\hline LPS (EU/mL) & $2.47 \pm 0.62$ & $1.84 \pm 0.40$ & $2.92 \pm 0.25$ & $<0.001$ \\
\hline OSAS n (\%) & $52(63 \%)$ & $5(16 \%)$ & $47(95 \%)$ & $<0.001$ \\
\hline Severe OSAS n (\%)‡ & $23(29 \%)$ & $0 \%$ & $23(47 \%)$ & $<0.001$ \\
\hline AHI (events/h) & $3.45 \pm 3.21$ & $0.89 \pm 0.81$ & $5.06 \pm 3.96$ & $<0.001$ \\
\hline ODI & $1.03 \pm 1.77$ & $0.73 \pm 0.72$ & $1.22 \pm 1.57$ & 0.030 \\
\hline TST (h) & $7.9 \pm 0.6$ & $7.8 \pm 0.8$ & $8.0 \pm 0.9$ & 0.238 \\
\hline Mean $\mathrm{SaO}_{2}(\%)$ & $95.9 \pm 1.0$ & $96.1 \pm 0.9$ & $95.9 \pm 1.1$ & 0.266 \\
\hline Nadir $\mathrm{SaO}_{2}(\%)$ & $88.5 \pm 1.8$ & $91.8 \pm 1.2$ & $86.5 \pm 1.8$ & $<0.001$ \\
\hline $\mathrm{SaO}_{2}<90 \%(\% \mathrm{TST})$ & $34.3 \pm 37.7$ & $18.7 \pm 18.6$ & $40.5 \pm 37.4$ & $<0.001$ \\
\hline $\mathrm{ETpCO}_{2}(\mathrm{~mm} \mathrm{Hg})$ & $47.3 \pm 6.5$ & $44.4 \pm 3.6$ & $49.1 \pm 7.1$ & $<0.001$ \\
\hline AST (IU/L) & $37 \pm 17$ & $33 \pm 15$ & $39 \pm 15$ & 0.085 \\
\hline ALT (IU/L) & $41 \pm 18$ & $34 \pm 16$ & $45 \pm 16$ & $\begin{array}{l}0.011 \\
0.004\end{array}$ \\
\hline GGT (IU/L) & $22 \pm 15$ & $17 \pm 7$ & $25 \pm 11$ & 0.001 \\
\hline CK18 (U/L) & $280 \pm 80$ & $195 \pm 36$ & $338 \pm 42$ & $<0.001$ \\
\hline Hyaluronic acid (ng/mL) & $1308 \pm 553$ & $1052 \pm 465$ & $1464 \pm 547$ & $<0.001$ \\
\hline
\end{tabular}

Data are reported as mean $\pm S D$, unless otherwise specified. Differences were considered statistically significant at $p<0.05$. Statistically significant differences are highlighted in bold. Pathological L/M ratio: an L/M ratio $\geq 0.03$ between fractional excretion of lactulose and the fractional excretion of mannitol was considered abnormal.

${ }^{*}$ Obesity: $\mathrm{BMI} \geq 95$ th percentile.

$\dagger$ Abdominal obesity: waist circumference $\geq 90$ th percentile for age and sex.

‡Severe OSAS: AHI $\geq 5$ events/h.

AHI (apnoea/hypopnoea index), number of apnoea/hypopnoeas per hour of sleep; ALT, alanine transaminase; AST, aspartate aminotransferase; BMI, body mass index; BP, blood pressure; CK18, cytokeratin-18 fragments; ETpCO 2 , mean end-tidal $\mathrm{PCO}_{2}$; GGT, gamma-glutamyl transpeptidase; HDL-C, high-density lipoprotein cholesterol; HOMA-IR, homeostasis model assessment of insulin resistance; IL, interleukin; ISI, insulin sensitivity index; LPS, lipopolysaccharide; NAFLD, non-alcoholic fatty liver disease; NASH, non-alcoholic steatohepatitis; ODI, oxygen desaturation index; OSAS, obstructive sleep apnoea syndrome; RBP, retinol-binding protein; $\mathrm{SaO}_{2}<90 \%$, total duration of haemoglobin desaturation, expressed as \% TST; TNF, tumour necrosis factor; TST, total sleep time (min). 
Table 2 Liver histology, immunohistochemistry and immunofluorescence of study population ( $\mathrm{n}=80$ ), grouped according to the presence/ absence of NASH

\begin{tabular}{|c|c|c|c|c|}
\hline Parameter & NAFLD $(n=80)$ & Not NASH $(n=31)$ & NASH $(n=49)$ & $\begin{array}{l}\text { P value } \\
\text { NASH versus not NASH }\end{array}$ \\
\hline Steatosis score & $2.3 \pm 0.7$ & $1.6 \pm 0.6$ & $2.6 \pm 0.5$ & $<0.001$ \\
\hline Lobular inflammatory score & $1.5 \pm 0.7$ & $1.1 \pm 0.5$ & $1.8 \pm 0.5$ & $<0.001$ \\
\hline Ballooning score & $1.1 \pm 0.6$ & $0.6 \pm 0.5$ & $1.4 \pm 0.6$ & $<0.001$ \\
\hline NAS & $4.9 \pm 1.4$ & $3.4 \pm 0.7$ & $5.7 \pm 0.8$ & $<0.001$ \\
\hline Fibrosis stage & $1.4 \pm 0.7$ & $0.9 \pm 0.7$ & $1.61 \pm 0.73$ & $<0.001$ \\
\hline \multicolumn{5}{|l|}{ Fibrosis stage $\mathrm{n}(\%)$} \\
\hline 0 & $10(13 \%)$ & $9(29 \%)$ & $1(2 \%)$ & 0.001 \\
\hline 1 & $35(44 \%)$ & $15(48 \%)$ & $20(41 \%)$ & 0.665 \\
\hline $1 a$ & $0 \%$ & $0 \%$ & $0 \%$ & 0.899 \\
\hline $1 b$ & $11(8 \%)$ & $5(16 \%)$ & $6(12 \%)$ & 0.813 \\
\hline $1 c$ & $24(30 \%)$ & $9(29 \%)$ & $15(31 \%)$ & 0.920 \\
\hline 2 & $32(40 \%)$ & $7(23 \%)$ & $25(51 \%)$ & 0.022 \\
\hline 3 & $3(8 \%)$ & $0 \%$ & $3(6 \%)$ & 0.424 \\
\hline 4 & $0 \%$ & $0 \%$ & $0 \%$ & 0.899 \\
\hline Fibrosis any stage $\mathrm{n}(\%)$ & $70(88 \%)$ & $22(71 \%)$ & $48(98 \%)$ & 0.001 \\
\hline Portal fibrosis n (\%) & $56(70 \%)$ & $16(52 \%)$ & $40(82 \%)$ & 0.009 \\
\hline Significant (stage $\geq 2$ ) fibrosis $n(\%)$ & $35(44 \%)$ & $7(23 \%)$ & $28(57 \%)$ & 0.005 \\
\hline \multicolumn{5}{|l|}{ Hepatocytes } \\
\hline Cell-cycle arrest index* & $7.89 \pm 6.94$ & $3.71 \pm 2.89$ & $12.07 \pm 6.35$ & $<0.001$ \\
\hline Apoptotic index* & $9.61 \pm 7.7$ & $5.36 \pm 6.63$ & $13.86 \pm 6.57$ & $<0.001$ \\
\hline Adiponectin-positive hepatocytes (\%) & $17.2 \pm 11.3$ & $23.5 \pm 10.4$ & $8.5 \pm 5.6$ & $<0.001$ \\
\hline Resistin-positive hepatocytes(\%) & $18.4 \pm 13.3$ & $20.1 \pm 12.8$ & $15.9 \pm 13.5$ & 0.171 \\
\hline TLR-4-positive hepatocytes (\%)† & $54.1 \pm 16.2$ & $39.0 \pm 5.9$ & $69.2 \pm 5.4$ & $<0.001$ \\
\hline \multicolumn{5}{|l|}{ Kupffer cells } \\
\hline n-Kupffer cellst & $9.8 \pm 3.8$ & $7.8 \pm 2.9$ & $11.9 \pm 3.7$ & $<0.001$ \\
\hline \multicolumn{5}{|l|}{ TLR-4-positive Kupffer cells } \\
\hline n-cells & $5.1 \pm 3.1$ & $2.9 \pm 2.2$ & $7.2 \pm 2.4$ & $<0.001$ \\
\hline$\%$ cells & $48.3 \pm 17.6$ & $35.5 \pm 14.9$ & $61.1 \pm 8.5$ & $<0.001$ \\
\hline \multicolumn{5}{|l|}{ HSCS } \\
\hline $\mathrm{n}$-HSCst & $5.9 \pm 1.9$ & $4.7 \pm 1.6$ & $7.0 \pm 1.6$ & $<0.001$ \\
\hline \multicolumn{5}{|l|}{ TLR-4-positive HSCs } \\
\hline n-cells & $2.4 \pm 1.3$ & $1.3 \pm 0.6$ & $3.4 \pm 0.8$ & $<0.001$ \\
\hline$\%$ total HSCs & $38.9 \pm 14.9$ & $28.2 \pm 10.8$ & $49.6 \pm 10.0$ & $<0.001$ \\
\hline \multicolumn{5}{|l|}{ HPCs } \\
\hline n-HPCs $\ddagger$ & $41.7 \pm 9.1$ & $37.6 \pm 8.3$ & $43.6 \pm 9.2$ & 0.004 \\
\hline \multicolumn{5}{|l|}{ Adiponectin-positive HPCs } \\
\hline $\mathrm{n}-\mathrm{HPCs}$ & $4.4 \pm 0.7$ & $5.3 \pm 0.7$ & $2.9 \pm 0.5$ & $<0.001$ \\
\hline$\% \mathrm{HPCs}$ & $10.5 \pm 8.1$ & $14.1 \pm 8.6$ & $6.8 \pm 5.7$ & $<0.001$ \\
\hline \multicolumn{5}{|l|}{ Resistin-positive HPCs } \\
\hline $\mathrm{n}$-HPCs & $8.9 \pm 0.9$ & $8.6 \pm 1.0$ & $9.0 \pm 1.1$ & 0.105 \\
\hline$\% \mathrm{HPCS}$ & $21.5 \pm 9.4$ & $22.3 \pm 8.6$ & $20.9 \pm 10.4$ & 0.533 \\
\hline Intermediate hepatocytes (\% patients) & $11(14 \%)$ & $0 \%$ & $11(22 \%)$ & 0.012 \\
\hline
\end{tabular}

Data are reported as mean $\pm S D$, unless otherwise specified. Statistically significant differences $(p<0.05)$ are highlighted in bold.

${ }^{*}$ At least 30 lobular fields at $\times 40$ magnification were analysed (1000 hepatocytes) for each section.

tMean count in five different fields at a magnification of $\times 20$ under light microscopy.

$\neq$ Cells per HPF, that is, at a magnification of $\times 20$ under light microscopy.

HPC, hepatic progenitor cells; HPF, high-power field; HSC, hepatic stellate cells; NAFLD, non-alcoholic fatty liver disease; NAS, NAFLD activity score; NASH, non-alcoholic steatohepatitis; TLR, toll-like receptor.

lesions (eg, zonality of lesions, portal inflammation and fibrosis). ${ }^{8} 13$

Liver immunofluorescence and immunohistochemistry Liver immunofluorescence (IF) and immunohistochemistry (IHC) were performed on 2 - $\mu$ m-thick sections obtained from formalin-fixed tissue embedded in paraffin. Antigen retrieval was performed with EDTA (pH 8) (Dako, Glostrup, Denmark).

Each case was analysed by IF for $\alpha$-smooth muscle actin ( $\alpha$-SMA), CK8/18, CD68 and TLR-4. For the identification of activated hepatic stellate cells (HSCs) and portal/septal myofibroblasts, the primary antibody used was anti- $\alpha$-SMA (dilution 1:200 overnight; mouse monoclonal, clone 1A4, Novus Biological, Littleton, Colorado, USA); for the detection of Kupffer cells and macrophages, the primary antibody used was anti-CD68 (dilution 1:200 overnight; mouse monoclonal, clone KP1, Abcam, Burlingame, California, USA); and for the detection of hepatocytes, the primary antibody used was CK8/ 18 (dilution 1:100 incubated for $1 \mathrm{~h}$, mouse monoclonal, Vector Laboratories, Burlingame, California, USA) (details provided in online supplementary file). 
Table 3 Clinical and biochemical features of study population, grouped according to the presence and severity of OSAS ( $n=80$ )

\begin{tabular}{|c|c|c|c|c|c|c|}
\hline Parameter & $\begin{array}{l}\text { Non-OSAS subjects } \\
(\mathrm{n}=28)\end{array}$ & $\begin{array}{l}\text { OSAS subjects } \\
(n=52)\end{array}$ & $\begin{array}{l}P \text { value } \\
\text { OSAS versus } \\
\text { non- OSAS }\end{array}$ & $\begin{array}{l}\text { Mild OSAS } \\
(n=29)\end{array}$ & $\begin{array}{l}\text { Severe OSAS } \\
(n=23)\end{array}$ & $\begin{array}{l}p \text { Value } \\
\text { mild versus severe } \\
\text { OSAS }\end{array}$ \\
\hline Age (years) & $11.7 \pm 1.9$ & $11.3 \pm 2.1$ & 0.469 & $11.4 \pm 1.9$ & $11.2 \pm 2.5$ & 0.759 \\
\hline Gender $\mathrm{n}(\%)$ males & $18(64 \%)$ & 27 (52\%) & 0.290 & 15 (52\%) & $12(52 \%)$ & 0.999 \\
\hline BMI $\left(\mathrm{kg} / \mathrm{m}^{2}\right)$ & $26.4 \pm 5.9$ & $28.3 \pm 4.9$ & 0.129 & $28.4 \pm 5.1$ & $28.5 \pm 5.0$ & 0.994 \\
\hline BMI z-score & $1.77 \pm 0.65$ & $2.04 \pm 0.68$ & 0.101 & $2.03 \pm 0.53$ & $2.09 \pm 0.48$ & 0.671 \\
\hline Obese subjects $n(\%)$ & $18(64 \%)$ & $41(79 \%)$ & 0.186 & $23(79 \%)$ & $18(78 \%)$ & 0.928 \\
\hline Waist circumference $(\mathrm{cm})$ & $89.0 \pm 5.4$ & $89.8 \pm 3.9$ & 0.492 & $90.0 \pm 4.5$ & $89.5 \pm 2.9$ & 0.676 \\
\hline $\begin{array}{l}\text { Waist circumference } \\
z \text {-score }\end{array}$ & $1.95 \pm 0.83$ & $2.01 \pm 0.76$ & 0.492 & $1.97 \pm 0.69$ & $2.09 \pm 0.86$ & 0.640 \\
\hline Systolic BP (mm Hg) & $113 \pm 16$ & $111 \pm 9$ & 0.569 & $111 \pm 10$ & $113 \pm 13$ & 0.613 \\
\hline Diastolic BP (mm Hg) & $67 \pm 8$ & $68 \pm 8$ & 0.540 & $68 \pm 8$ & $69 \pm 8$ & 0.892 \\
\hline Insulin $(\mu \mathrm{U} / \mathrm{mL})$ & $15.1 \pm 10.7$ & $13.1 \pm 9.1$ & 0.381 & $15.0 \pm 9.6$ & $11.6 \pm 9.4$ & 0.213 \\
\hline Glucose (mg/dL) & $87 \pm 15$ & $85 \pm 15$ & 0.459 & $83 \pm 11$ & $87 \pm 16$ & 0.316 \\
\hline HOMA-IR & $3.14 \pm 2.10$ & $2.88 \pm 2.02$ & 0.460 & $3.11 \pm 2.16$ & $2.49 \pm 2.10$ & 0.213 \\
\hline ISI & $3.55 \pm 2.57$ & $2.64 \pm 1.99$ & 0.110 & $2.66 \pm 1.60$ & $2.65 \pm 1.39$ & 0.962 \\
\hline Triglyceride (mg/dL) & $105 \pm 51$ & $116 \pm 75$ & 0.409 & $127 \pm 83$ & $108 \pm 56$ & 0.305 \\
\hline Total cholesterol (mg/dL) & $159 \pm 28$ & $163 \pm 37$ & 0.337 & $167 \pm 42$ & $160 \pm 34$ & 0.582 \\
\hline $\mathrm{HDL}-\mathrm{C}(\mathrm{mg} / \mathrm{dL})$ & $43 \pm 7$ & $42 \pm 7$ & 0.468 & $40 \pm 6$ & $43 \pm 7^{*}$ & 0.127 \\
\hline Diabetes n (\%) & $1(4 \%)$ & $1(2 \%)$ & 0.812 & $0 \%$ & $1(4 \%)$ & 0.613 \\
\hline Metabolic syndrome $\mathrm{n}(\%)$ & $3(11 \%)$ & $10(19 \%)$ & 0.505 & $6(21 \%)$ & $4(17 \%)^{*}$ & 0.591 \\
\hline Abdominal obesity $\mathrm{n}(\%)$ & $19(67 \%)$ & $31(60 \%)$ & 0.891 & $19(66 \%)$ & $12(52 \%)$ & 0.602 \\
\hline Hypertension $\mathrm{n}(\%)$ & $5(19 \%)$ & $6(13 \%)$ & 0.694 & $2(7 \%)$ & $4(17 \%)$ & 0.191 \\
\hline Hypertriglyceridemia n (\%) & $6(21 \%)$ & $16(31 \%)$ & 0.271 & $12(41 \%)$ & $4(17 \%)$ & 0.119 \\
\hline Low HDL-C n (\%) & $9(32 \%)$ & $13(25 \%)$ & 0.513 & $10(34 \%)$ & $3(13 \%)$ & 0.147 \\
\hline IFG/diabetes n (\%) & $5(18 \%)$ & $9(17 \%)$ & 0.998 & $5(17 \%)$ & $4(17 \%)$ & 0.479 \\
\hline Adiponectin (ng/mL) & $22.7 \pm 2.4$ & $20.9 \pm 1.8$ & 0.014 & $21.0 \pm 2.0$ & $20.7 \pm 1.4$ & 0.513 \\
\hline Resistin (pg/mL) & $29886 \pm 11991$ & $36921 \pm 29326$ & 0.554 & $37102 \pm 32012$ & $35112 \pm 10940$ & 0.890 \\
\hline RBP-4 (mg/mL) & $2.9 \pm 1.1$ & $3.3 \pm 1.3$ & 0.171 & $3.26 \pm 1.87$ & $3.33 \pm 1.25$ & 0.867 \\
\hline C-reactive protein (mg/L) & $1.6 \pm 0.4$ & $1.5 \pm 0.4$ & 0.763 & $1.6 \pm 0.4$ & $1.5 \pm 0.5$ & 0.661 \\
\hline IL-6 (pg/mL) & $11.0 \pm 5.6$ & $11.5 \pm 5.9$ & 0.759 & $11.8 \pm 6.4$ & $10.5 \pm 4.3$ & 0.226 \\
\hline Leptin (ng/mL) & $18.9 \pm 7.7$ & $19.5 \pm 9.6$ & 0.755 & $18.9 \pm 9.7$ & $22.3 \pm 8.1$ & 0.567 \\
\hline TNF- $\alpha(p g / m L)$ & $6.8 \pm 2.1$ & $6.6 \pm 2.2$ & 0.756 & $6.5 \pm 2.3$ & $7.2 \pm 2.1$ & 0.549 \\
\hline L/M ratio & $0.022 \pm 0.027$ & $0.059 \pm 0.040$ & $<0.001$ & $0.041 \pm 0.030$ & $0.088 \pm 0.045$ & $<0.001$ \\
\hline Pathological L/M ratio (\%) & $2(7 \%)$ & $26(50 \%)$ & $<0.001$ & $10(34 \%)$ & $16(69 \%)$ & 0.026 \\
\hline LPS (EU/mL) & $1.97 \pm 0.52$ & $2.83 \pm 0.41$ & $<0.001$ & $2.70 \pm 0.20$ & $2.98 \pm 0.19$ & 0.010 \\
\hline AHI (events/h) & $0.58 \pm 0.30$ & $4.99 \pm 3.07$ & $<0.001$ & $2.15 \pm 1.21 \dagger$ & $8.77 \pm 3.05 \dagger$ & $<0.001$ \\
\hline ODI & $0.62 \pm 0.60$ & $1.45 \pm 1.11$ & 0.035 & $0.91 \pm 0.64 \ddagger$ & $2.14 \pm 1.00 \dagger$ & 0.009 \\
\hline TST (h) & $7.8 \pm 0.8$ & $8.0 \pm 0.7$ & 0.252 & $8.1 \pm 0.8$ & $7.9 \pm 0.7$ & 0.305 \\
\hline Mean $\mathrm{SaO}_{2}(\%)$ & $96.4 \pm 0.9$ & $94.6 \pm 0.8$ & $<0.001$ & $93.7 \pm 0.7^{*}$ & $92.0 \pm 0.8 \ddagger$ & 0.022 \\
\hline Nadir $\mathrm{SaO}_{2}(\%)$ & $90.8 \pm 1.1$ & $86.4 \pm 1.3$ & $<0.001$ & $88.0 \pm 1.6 \ddagger$ & $84.5 \pm 1.2 \dagger$ & 0.011 \\
\hline $\mathrm{SaO}_{2}<90 \%$ (\%TST) & $12 \pm 13$ & $46 \pm 21$ & $<0.001$ & $40 \pm 15 \dagger$ & $55 \pm 18 \dagger$ & $<0.001$ \\
\hline $\mathrm{ETpCO}_{2}(\mathrm{~mm} \mathrm{Hg})$ & $44.0 \pm 3.5$ & $48.9 \pm 7.0$ & $<0.001$ & $47.1 \pm 4.3$ & $52.0 \pm 7.8$ & 0.028 \\
\hline AST (IU/L) & $33 \pm 16$ & $37 \pm 18$ & 0.295 & $32 \pm 13$ & $44 \pm 18$ & 0.049 \\
\hline ALT (IU/L) & $34 \pm 15$ & $41 \pm 15$ & 0.081 & $38 \pm 17$ & $42 \pm 16$ & 0.744 \\
\hline GGT (IU/L) & $18 \pm 6$ & $21 \pm 16$ & 0.229 & $21 \pm 17$ & $21 \pm 12$ & 0.813 \\
\hline CK-18 (U/L) & $208 \pm 54$ & $301 \pm 62$ & $<0.001$ & $291 \pm 55$ & $339 \pm 46$ & 0.007 \\
\hline Hyaluronic acid (ng/mL) & $1124 \pm 455$ & $1401 \pm 466$ & 0.009 & $1224 \pm 541$ & $1626 \pm 528 \ddagger$ & 0.005 \\
\hline
\end{tabular}

Data are reported as mean $\pm S D$, unless otherwise specified. Differences were considered statistically significant at $p<0.05$. Statistically significant differences are highlighted in bold. Severe OSAS: AHI $\geq 5$ events/h. Obesity, BMI $\geq 95$ th percentile.

${ }^{*} p<0.05$ versus non-OSAS subjects.

$t<0.0001$ versus non-OSAS subjects.

$\neq \mathrm{p}<0.01$ versus non-OSAS subjects.

AHI (apnoea/hypopnoea index, number of apnoea/hypopnoeas per hour of sleep; ALT, alanine transaminase; AST, aspartate aminotransferase; BMI, body mass index; BP, blood pressure; $\mathrm{CK} 18$, cytokeratin-18 fragments; $\mathrm{ETPCO}_{2}$, mean end-tidal $\mathrm{pCO}$; GGT, gamma-glutamyl transpeptidase; HOMA-IR, homeostasis model assessment of insulin resistance; IL, interleukin; ISI, insulin sensitivity index; LPS, lipopolysaccharide; ODI, oxygen desaturation index; OSAS, obstructive sleep apnoea syndrome; pathological L/M ratio, an L/M ratio $\geq 0.03$ between

fractional excretion of lactulose and the fractional excretion of mannitol was considered abnormal; RBP, retinol-binding protein; $\mathrm{SaO}_{2}<90 \%$, total duration of haemoglobin desaturation, expressed as \%TST; TNF, tumour necrosis factor; TST, total sleep time (min). 
Table 4 Liver histology, immunohistochemistry and immunofluorescence of study population ( $\mathrm{n}=80$ ), grouped according to the presence and severity of OSAS

\begin{tabular}{|c|c|c|c|c|c|c|}
\hline Parameter & $\begin{array}{l}\text { Non-OSAS } \\
\text { subjects } \\
(n=28)\end{array}$ & $\begin{array}{l}\text { OSAS } \\
\text { subjects } \\
(n=52)\end{array}$ & $\begin{array}{l}\text { p Value } \\
\text { OSAS versus } \\
\text { non- OSAS }\end{array}$ & $\begin{array}{l}\text { Mild OSAS } \\
(n=29)\end{array}$ & $\begin{array}{l}\text { Severe } \\
\text { OSAS } \\
(n=23)\end{array}$ & $\begin{array}{l}\text { p Value } \\
\text { mild versus } \\
\text { severe OSAS }\end{array}$ \\
\hline NASH n (\%) & $2(7 \%)$ & $47(90 \%)$ & $<0.001$ & $24(83 \%)^{*}$ & $23(100 \%)^{*}$ & 0.105 \\
\hline Steatosis score & $1.7 \pm 0.5$ & $2.5 \pm 0.6$ & $<0.001$ & $2.3 \pm 0.4^{*}$ & $2.7 \pm 0.5^{*}$ & 0.007 \\
\hline Lobular inflammatory score & $1.1 \pm 0.6$ & $1.8 \pm 0.5$ & $<0.001$ & $1.5 \pm 0.4 \dagger$ & $1.8 \pm 0.5^{*}$ & 0.026 \\
\hline Ballooning score & $0.8 \pm 0.5$ & $1.3 \pm 0.6$ & $<0.001$ & $1.1 \pm 0.3+$ & $1.4 \pm 0.4^{*}$ & 0.001 \\
\hline NAS & $3.6 \pm 0.8$ & $5.5 \pm 1.1$ & $<0.001$ & $4.7 \pm 1.0^{*}$ & $5.8 \pm 0.9^{*}$ & $<0.001$ \\
\hline Fibrosis stage & $1.1 \pm 0.6$ & $1.5 \pm 0.7$ & 0.009 & $1.3 \pm 0.6$ & $1.7 \pm 0.6 \ddagger$ & 0.027 \\
\hline \multicolumn{7}{|l|}{ Subjects with fibrosis stage $\mathrm{n}(\%)$} \\
\hline 0 & $10(32 \%)$ & $0 \%$ & $<0.001$ & $0(0 \%)$ & $0 \%$ & 0.673 \\
\hline 1 & $13(43 \%)$ & $22(44 \%)$ & 0.906 & $22(76 \%)$ & $0 \%$ & $<0.001$ \\
\hline $1 \mathrm{a}$ & $0 \%$ & $0 \%$ & 0.851 & $0 \%$ & $0 \%$ & 0.673 \\
\hline $1 b$ & $5(18 \%)$ & $5(12 \%)$ & 0.478 & $5(17 \%)$ & $0 \%$ & 0.097 \\
\hline $1 c$ & $8(32 \%)$ & $17(29 \%)$ & 0.899 & $17(59 \%)$ & $0 \%$ & $<0.001$ \\
\hline 2 & $5(14 \%)$ & $27(54 \%)$ & 0.006 & $7(24 \%)$ & $20(87 \%) \ddagger$ & $<0.001$ \\
\hline 3 & $0 \%$ & $3(6 \%)$ & 0.612 & $0(\%)$ & $3(13 \%)$ & 0.160 \\
\hline 4 & $0 \%$ & $0 \%$ & 0.999 & $0 \%$ & $0 \%$ & 0.978 \\
\hline Fibrosis any stage $n(\%)$ & $18(64 \%)$ & $52(100 \%)$ & $<0.001$ & $29(100 \%)$ & $23(100 \%)$ & 0.987 \\
\hline Portal fibrosis n (\%) & $13(46 \%)$ & $44(85 \%)$ & $<0.001$ & $24(83 \%)$ & $20(87 \%)$ & 0.942 \\
\hline Significant $(F \geq 2)$ fibrosis $n(\%)$ & $5(18 \%)$ & $30(58 \%)$ & 0.001 & $7(24 \%)$ & $23(100 \%) \ddagger^{*}$ & $<0.001$ \\
\hline \multicolumn{7}{|l|}{ Hepatocytes } \\
\hline Cell-cycle arrest index§ & $4.68 \pm 3.98$ & $11.01 \pm 6.04$ & $<0.001$ & $8.97 \pm 6.13 \dagger$ & $15.01 \pm 8.11^{*}$ & 0.004 \\
\hline Apoptotic index§ & $6.71 \pm 6.54$ & $12.50 \pm 7.14$ & $<0.001$ & $9.01 \pm 4.58 t$ & $16.58 \pm 6.13$ & $<0.001$ \\
\hline Adiponectin-positive hepatocytes (\%) & $23.5 \pm 10.3$ & $8.5 \pm 5.5$ & $<0.001$ & $12.4 \pm 5.1 \dagger$ & $5.8 \pm 3.9^{*}$ & $<0.001$ \\
\hline Resistin-positive hepatocytes (\%) & $20.1 \pm 12.8$ & $15.9 \pm 13.4$ & 0.207 & $15.8 \pm 12.1$ & $13.9 \pm 13.5$ & 0.704 \\
\hline TLR-4-positive hepatocytes (\%)ף & $41.6 \pm 7.4$ & $63.3 \pm 14.8$ & $<0.001$ & $59.1 \pm 11.3^{*}$ & $69.8 \pm 6.1^{*}$ & $<0.001$ \\
\hline \multicolumn{7}{|l|}{ Kupffer cells } \\
\hline n-Kupffer cells & $8.0 \pm 3.4$ & $11.2 \pm 3.7$ & $<0.001$ & $10.7 \pm 2.5 \ddagger^{*}$ & $12.7 \pm 1.9^{*}$ & 0.003 \\
\hline \multicolumn{7}{|l|}{ TLR-4-positive Kupffer cells } \\
\hline n-cells & $3.1 \pm 2.4$ & $6.6 \pm 2.8$ & $<0.001$ & $5.7 \pm 2.8+$ & $7.2 \pm 1.9^{*}$ & 0.033 \\
\hline$\%$ cells & $36.9 \pm 15.4$ & $57.4 \pm 13.1$ & $<0.001$ & $50.1 \pm 12.1 \dagger$ & $65.4 \pm 9.1^{*}$ & $<0.001$ \\
\hline \multicolumn{7}{|l|}{ HSCs } \\
\hline $\mathrm{n}$-HSCsף & $5.0 \pm 1.4$ & $6.4 \pm 1.6$ & $<0.001$ & $6.0 \pm 1.6 \ddagger^{*}$ & $7.1 \pm 1.7 \dagger$ & 0.020 \\
\hline \multicolumn{7}{|l|}{ TLR-4-positive HSCs } \\
\hline$n-H S C$ & $1.4 \pm 0.6$ & $3.1 \pm 1.1$ & $<0.001$ & $2.4 \pm 0.7 \ddagger^{*}$ & $3.7 \pm 0.9^{*}$ & $<0.0010$ \\
\hline$\%$ HSCs & $27.1 \pm 10.5$ & $48.4 \pm 11.1$ & $<0.001$ & $40.1 \pm 10.5 t$ & $52.1 \pm 11.4^{*}$ & $<0.001$ \\
\hline \multicolumn{7}{|l|}{ HPCs } \\
\hline $\mathrm{n}-\mathrm{HPCs}$ ** & $37.1 \pm 8.7$ & $44.1 \pm 8.9$ & 0.001 & $40.0 \pm 8.2 \ddagger^{*}$ & $46.7 \pm 8.1 \dagger$ & 0.005 \\
\hline \multicolumn{7}{|l|}{ Adiponectin-positive } \\
\hline $\mathrm{n}$ HPCs & $5.2 \pm 3.0$ & $3.3 \pm 2.1$ & 0.001 & $3.8 \pm 1.7 \dagger$ & $2.8 \pm 1.2^{*}$ & 0.021 \\
\hline$\%$ HPCs & $13.9 \pm 8.2$ & $7.5 \pm 5.7$ & $<0.001$ & $9.5 \pm 5.1 \dagger$ & $6.4 \pm 4.9^{*}$ & 0.031 \\
\hline \multicolumn{7}{|l|}{ Resistin-positive } \\
\hline n HPCs & $8.5 \pm 3.0$ & $8.9 \pm 4.1$ & 0.651 & $9.0 \pm 3.8$ & $9.5 \pm 4.0$ & 0.647 \\
\hline$\%$ HPCs & $23.0 \pm 8.5$ & $20.2 \pm 10.4$ & 0.463 & $22.5 \pm 9.7$ & $20.4 \pm 8.9$ & 0.425 \\
\hline Intermediate hepatocytes (\% patients) & $0 \%$ & $11(21 \%)$ & 0.023 & $2(7 \%)$ & $9(39 \%) \ddagger^{*}$ & 0.034 \\
\hline
\end{tabular}

\footnotetext{
Data are reported as mean \pm SD, unless otherwise specified. Differences were considered statistically significant at $p<0.05$. Statistically significant differences are highlighted in bold. ${ }^{*} p<0.0001$ versus non-OSAS subjects.

Double CD68/TLR-4 and $\alpha$-SMA/TLR-4-positive cells were manually counted in at least 10 fields at $\times 20$ magnification for each sample. Data were expressed both as a percentage of positive cells. The intensity average of TLR-4 fluorescence in CK8/ 18-positive hepatocytes was assessed in at least 200 regions of interest for sample using MetaMorph software.

Observations were processed and images were analysed by two independent pathologists blinded to the patient's data. 


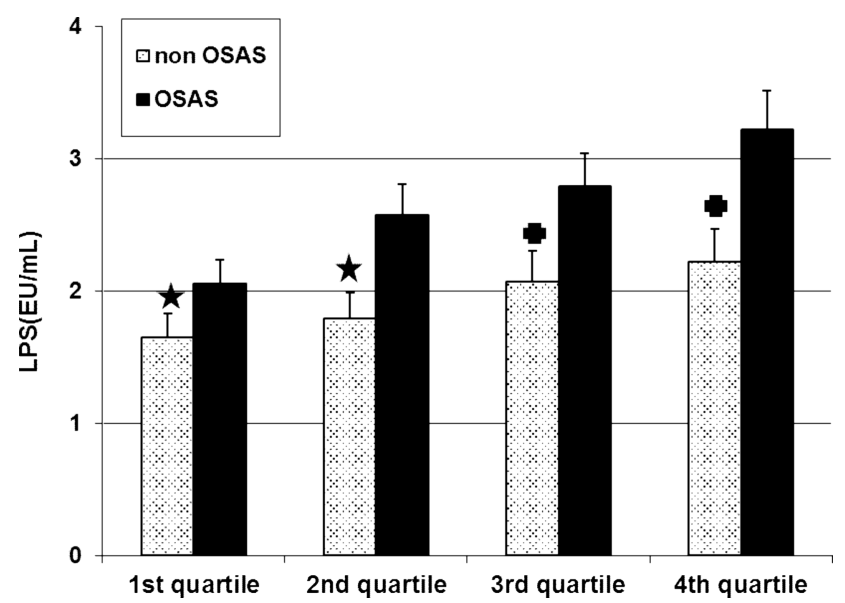

Figure 1 Impact of the presence of obstructive sleep apnoea syndrome (OSAS) on plasma lipopolysaccharide (LPS) within the same quartile of body mass index (BMI) $z$-score. Data are expressed as mean \pm SD. $\star p<0.05$ versus OSAS within the same quartile of BMI $z$-score. - $p<0.01$ versus OSAS within the same quartile of BMI $z$-score.
Adipokine expression by HPCs

The primary antibody against CK7 (dilution 1/200; mouse monoclonal, clone OV-TL 12/30, Dako) was used for the identification of HPCs as previously described by Roskams et al. ${ }^{26}$

For IHC, sections were incubated overnight at $4^{\circ} \mathrm{C}$ with primary antibodies against $\mathrm{CK} 7$, adiponectin, resistin, p2 $21^{\text {Waf1 }}$ and cleaved caspase-3 (primary antibodies are listed in online supplementary table S1).

The number of HPCs within the ductular reaction was counted within the entire section and expressed as the number of CK7-positive cells per high-power field (HPF; at $\times 20)$.

Intermediate hepatocytes (IHs) were defined as cells with sizes between those of hepatocytes and HPCs $(<40$ but $>6 \mu \mathrm{m}$ in diameter), with faint CK7 immunoreactivity in the cytoplasm and reinforcement at the plasma membrane. Based on our recent data, IHs were scored as present or absent. $^{12}$

Adiponectin and resistin expression by CK7-positive HPCs was evaluated in serial sections and confirmed with IF. Data were expressed both as number of positive cells per HPF and as

Table 5 Predictors of the severity of liver histology on univariable and multivariable standard logistic regression analysis in the whole NAFLD population $(n=80)$

\begin{tabular}{|c|c|c|c|c|c|c|}
\hline \multirow[b]{2}{*}{ Parameter } & \multicolumn{3}{|c|}{ Univariable } & \multicolumn{3}{|c|}{ Multivariable } \\
\hline & OR & $95 \% \mathrm{Cl}$ & $p$ Value & OR & $95 \% \mathrm{Cl}$ & p Value \\
\hline \multicolumn{7}{|l|}{ Predictors of NASH } \\
\hline $\mathrm{SaO}_{2}<90 \%$ (\%TST) & 2.74 & 2.06 to 9.86 & $<0.001$ & 1.57 & 1.03 to 4.93 & 0.039 \\
\hline Plasma LPS (EU/mL) & 3.13 & 2.10 to 8.96 & $<0.001$ & 1.79 & 1.05 to 4.48 & 0.011 \\
\hline TLR-4-positive hepatocytes (\%) & 3.26 & 2.12 to 6.24 & $<0.001$ & 1.63 & 1.06 to 3.12 & 0.018 \\
\hline TLR-4-positive Kupffer cells (n) & 3.24 & 2.22 to 9.44 & $<0.001$ & 1.85 & 1.11 to 4.72 & 0.009 \\
\hline BMI z-score & 2.76 & 1.28 to 7.86 & 0.008 & - & - & - \\
\hline Plasma adiponectin (ng/mL) & 0.32 & 0.22 to 0.79 & 0.010 & - & - & - \\
\hline L/M ratio & 2.10 & 1.12 to 6.39 & 0.010 & - & - & - \\
\hline $\mathrm{ETpCO}_{2}(\mathrm{~mm} \mathrm{Hg})$ & 2.16 & 1.34 to 9.81 & 0.011 & - & - & - \\
\hline Hepatocyte cell-cycle arrest index (\%) & 2.56 & 1.64 to 9.41 & 0.009 & - & - & - \\
\hline Hepatocyte apoptotic index (\%) & 2.82 & 1.51 to 10.16 & 0.006 & - & - & - \\
\hline Adiponectin-positive HPCs (\%) & 0.43 & 1.18 to 8.13 & 0.021 & - & - & - \\
\hline \multicolumn{7}{|l|}{ Predictors of portal fibrosis } \\
\hline Plasma LPS (EU/mL) & 2.61 & 2.01 to 7.12 & $<0.001$ & 1.49 & 1.05 to 4.89 & 0.029 \\
\hline TLR-4-positive HSCs (n) & 3.20 & 2.20 to 6.11 & $<0.001$ & 1.83 & 1.12 to 3.05 & 0.015 \\
\hline Adiponectin-positive HPCs (n) & 0.41 & 0.22 to 0.73 & $<0.001$ & 0.88 & 0.81 to 0.98 & 0.041 \\
\hline BMI $z$-score & 2.06 & 1.63 to 12.83 & 0.001 & - & - & - \\
\hline $\mathrm{SaO}_{2}<90 \%(\% \mathrm{TST})$ & 2.12 & 1.64 to 5.93 & 0.002 & - & - & - \\
\hline Hepatic TLR-4-positive Kupffer cells (n) & 2.08 & 1.29 to 7.12 & 0.012 & - & - & - \\
\hline HPCs (n) & 2.82 & 1.17 to 11.52 & 0.029 & - & - & - \\
\hline NAS & 1.92 & 1.11 to 6.49 & 0.036 & - & - & - \\
\hline \multicolumn{7}{|l|}{ Predictors of significant (stage $\geq 2$ ) fibrosis } \\
\hline $\mathrm{SaO}_{2}<90 \%$ (\%TST) & 2.82 & 2.12 to 6.73 & $<0.001$ & 1.61 & 1.04 to 3.80 & 0.041 \\
\hline Plasma LPS (EU/mL) & 6.02 & 3.11 to 9.48 & $<0.001$ & 3.23 & 1.52 to 8.58 & 0.011 \\
\hline TLR-4-positive HSCs (n) & 5.65 & 2.94 to 9.93 & $<0.001$ & 3.80 & 1.49 to 5.32 & 0.005 \\
\hline BMI $z$-score & 2.13 & 1.19 to 8.93 & 0.012 & - & - & - \\
\hline Hepatic TLR-4-positive Kupffer cells (n) & 2.42 & 1.39 to 5.98 & 0.010 & - & - & - \\
\hline HPCs (n) & 2.89 & 1.38 to 6.93 & 0.009 & - & - & - \\
\hline Adiponectin-positive HPCs (n) & 0.73 & 0.46 to 0.91 & 0.034 & - & - & - \\
\hline NAFLD activity score (NAS) & 2.98 & 1.26 to 8.94 & 0.018 & - & - & - \\
\hline
\end{tabular}

The presence of $\mathrm{NASH}$, of portal and of significant (stage $\mathrm{F} \geq 2$ ) fibrosis were entered as dichotomous outcome variables (present/absent) into a multivariate logistic regression model. $\mathrm{AHI}$, apnoea/hypopnoea index; BMI, body mass index; $\mathrm{ETpCO}_{2}$, mean end-tidal $\mathrm{pCO}_{2}$; HPC, hepatic progenitor cell; HSC, hepatic stellate cell; L/M ratio, lactulose/mannitol ratio; LPS, lipopolysaccharide; NAFLD, non-alcoholic fatty liver disease; NAS, NAFLD activity score; NASH, non-alcoholic steatohepatitis; $0 D I$, oxygen desaturation index; SaO 2 < $90 \%$, total duration of haemoglobin desaturation, expressed as \%TST; TLR-4, toll-like receptor-4. 
a percentage of positive cells. Adipokine expression by hepatocytes was semiquantitatively evaluated and expressed as a percentage of positive cells.

\section{Hepatocyte apoptosis and cell-cycle arrest}

Apoptosis and cell-cycle arrest were assessed by counting the number of hepatocytes that stained strongly positive for cleaved caspase-3 (at the cytoplasm level) and p21 ${ }^{\text {waf1 }}$ (at the nuclear level). The apoptotic and p21 indices were calculated by dividing the average number of positive cells by the average number of hepatocytes and expressing the quotient as a percentage for each section. At least 30 lobular fields at $\times 40$ magnification were analysed $(\approx 1000$ hepatocytes) for each section.

\section{PSG study}

All biopsy-proven patients with NAFLD underwent an overnight PSG using standard techniques.

The patients received no dietary or lifestyle counselling or any other treatment between liver biopsy and PSG, which was performed within 3 months of the histological diagnosis of NAFLD.

PSG was carried out in a quiet room in the sleep laboratory of our hospital; all recordings started at the patients' usual bedtime and continued until spontaneous awakening.

No hypnotic drugs were allowed for at least two weeks before sleep recording. All patients were accompanied by one of their parents throughout the night. No oxygen was supplemented or respiratory stimulants were used.

The PSG montage included four EEG channels C3-A2, C4-A1, O1-A2 and O2-A1, left and right electrooculogram, chin electromyogram, ECG, nasal cannula, thoracic and abdominal respiratory effort, oxygen saturation (Siesta, Compumedics, Abbottsford, Australia), and end-tidal $\mathrm{pCO}_{2}\left(\mathrm{ETpCO}_{2}\right)$ was monitored simultaneously with other parameters (Capnostream, Oridion).

All recordings were manually and visually scored and interpreted according to current guidelines (2007 AASM Manual for the Scoring of Sleep and Associated Events: Rules, Terminology and Technical Specifications V.2.0). ${ }^{27}$

Central, obstructive and mixed apnoea events were counted according to the criteria established by the American Thoracic Society (2007): an obstructive apnoea was defined as the absence of airflow, with continued chest wall and abdominal movement, for a duration of at least two breaths; a central apnoea was defined as the absence of airflow with the cessation of respiratory effort, lasting $>20 \mathrm{~s}$ and associated with bradycardia and desaturation; central apnoea occurring after gross body movements or after sighs was not considered as a pathological finding; a mixed apnoea was defined as an apnoea that usually begins as central and ends in obstruction according to changes in the chest, abdominal and flow traces; hypopnoea was defined as a decrease in nasal flow of at least 50\% with a corresponding decrease in $\mathrm{SaO}_{2}$ of at least $4 \%$ and/or an arousal; the apnoea/ hypopnoea index (AHI) was defined as the number of apnoeas and hypopnoeas per hour of total sleep time (TST). Patients with an AHI $\geq 1$ event/h were considered to have OSAS, while subjects with an $\mathrm{AHI} \geq 5$ events/h were considered to have severe OSAS, according to current guidelines. ${ }^{10} 26$ The following parameters were also recorded: oxygen desaturation index, that is, the number of haemoglobin desaturations (drop in $\mathrm{SaO}_{2}$ $\geq 4 \%$ of baseline value) per hour of sleep, mean $\mathrm{SaO}_{2}$, nadir $\mathrm{SaO}_{2}$, total duration of haemoglobin desaturation $\left(\mathrm{SaO}_{2}\right.$ $<90 \%$ ), expressed as \%TST and mean $\mathrm{ETpCO}_{2}$.

The presence of adenotonsillar hypertrophy was assessed and graded according to a standard four-stage scale (from $0=$ absent to $4=$ severe), and other OSAS-related symptoms/signs were recorded at the time of PSG, as indicated by current guidelines and previously described. ${ }^{6} 10$

\section{Statistical analysis}

Sample size calculation: Based on limited available data on OSAS, ${ }^{8} 9$ parameters related to gut-liver axis ${ }^{11}$ and $\mathrm{HPC}^{13}$ in paediatric NAFLD, at least 74 subjects were needed to detect a significant $(p<0.05)$ difference in LPS, intestinal permeability, HPC number and hepatic adipokine expression between patients with and without OSAS with a power of $80 \%$.

Data were expressed as mean \pm SD. Differences were considered statistically significant at $\mathrm{p}<0.05$.

Differences across groups were analysed by analysis of variance and Bonferroni correction, when variables were normally distributed; otherwise, the Kruskal-Wallis test, followed by the post hoc Dunn test, was used to compare non-parametric variables. Normality was evaluated by Shapiro-Wilk test. $\chi^{2}$ test or Fisher's exact test was used to compare categorical variables, as appropriate. Spearman's rank correlation coefficient was used to estimate the relationship between different variables.

Multivariable standard logistic regression analysis was used to identify independent predictors of NASH, portal fibrosis and significant (stage $\geq 2$ ) fibrosis, included as a dichotomous variable. For this analysis, quartiles of continuous variables were included. The variables significantly associated with selected outcomes on univariable analysis were entered in each model. Multiple linear regression analysis was applied to identify predictors of NAS and fibrosis score and of different continuous variables. Continuous variables with skewed distribution were log-transformed. STATISTICA 5.1 (StatSoft Italia, Padua) was used for all analyses.

\section{RESULTS}

\section{Anthropometric, dietary and laboratory characteristics}

In total, 80 out of 81 children assented to participate to the study. Clinical, laboratory and histological data of the included children are described in tables 1 and 2 . In the whole NAFLD cohort, $73 \%$ subjects were obese and $16 \%$ had MS, $61 \%$ had $\mathrm{NASH}$ and $44 \%$ had significant $(\mathrm{F} \geq 2)$ fibrosis. No patient had cirrhosis. Compared with patients without NASH, children with $\mathrm{NASH}$ had a higher BMI, higher intestinal permeability and LPS levels and lower plasma adiponectin levels and a higher prevalence of OSAS (table 1). Polysomnographic parameters showed a skewed distribution and were all log-transformed for correlative analyses.

Dietary intake did not differ between NASH and non-NASH subjects and between OSAS and non-OSAS subjects and included a $40-45 \mathrm{cal} / \mathrm{kg} / \mathrm{day}$, derived from carbohydrate (50$60 \%)$, fat $(23-30 \%$, constituted of two-thirds unsaturated fatty acids and one-third saturated fatty acids) and protein (15-20\%).

Overall, the features of children included in this study resembled those seen in the general paediatric NAFLD population seen at our center. ${ }^{8} 11$

NASH is associated with increased hepatic TLR-4 expression and expansion of adiponectin-defective HPC compartment On liver IHC/IF, NASH was associated with an increased expression of TLR-4 by hepatocytes, Kupffer cells and HSCs, with increased hepatocyte apoptosis and cell-cycle arrest, and with an expansion of HPC compartment, coupled with a defective adiponectin expression by hepatocytes and HPCs (table 2 and online supplementary figure S1). 
Table 6 Predictors of the severity of different histological features on univariable and multivariable linear regression analysis in the whole NAFLD population $(n=80)$

\begin{tabular}{|c|c|c|c|c|c|c|}
\hline & \multicolumn{3}{|c|}{ Univariable } & \multicolumn{3}{|c|}{ Multivariable } \\
\hline & $\boldsymbol{\beta}$ & SE ( $(\beta)$ & $p$ Value & $\boldsymbol{\beta}$ & SE ( $(\boldsymbol{\beta})$ & p Value \\
\hline \multicolumn{7}{|l|}{ Predictors of steatosis score } \\
\hline $\mathrm{SaO}_{2}<90 \%(\% \mathrm{TST})$ & 0.593 & 0.113 & $<0.001$ & 0.339 & 0.101 & $<0.001$ \\
\hline TLR-4-positive hepatocytes (\%) & 0.601 & 0.123 & $<0.001$ & 0.345 & 0.127 & 0.008 \\
\hline L/M ratio & 0.235 & 0.139 & 0.042 & - & - & - \\
\hline Plasma adiponectin & -0.348 & 0.120 & 0.011 & - & - & - \\
\hline Plasma LPS & 0.426 & 0.169 & 0.009 & - & - & - \\
\hline $\mathrm{AHI}$ & 0.434 & 0.131 & 0.010 & - & - & - \\
\hline Nadir $\mathrm{SaO}_{2}(\%)$ & -0.228 & 0.117 & 0.032 & - & - & - \\
\hline Adiponectin-positive hepatocytes(\%) & -0.346 & 0.139 & 0.019 & - & - & - \\
\hline \multicolumn{7}{|l|}{ Predictors of hepatocyte ballooning } \\
\hline Plasma LPS & 0.611 & 0.109 & $<0.001$ & 0.341 & 0.113 & 0.009 \\
\hline $\mathrm{SaO}_{2}<90 \%$ (\%TST) & 0.682 & 0.141 & $<0.001$ & 0.364 & 0.145 & 0.002 \\
\hline TLR-4-positive hepatocytes (\%) & 0.694 & 0.173 & $<0.001$ & 0.449 & 0.124 & $<0.001$ \\
\hline Hepatocyte apoptotic index & 0.298 & 0.112 & 0.039 & - & - & - \\
\hline TLR-4-positive Kupffer cells (n) & 0.312 & 0.179 & 0.031 & - & - & - \\
\hline \multicolumn{7}{|l|}{ Predictors of lobular inflammation } \\
\hline Plasma LPS & 0.587 & 0.138 & $<0.001$ & 0.438 & 0.122 & 0.001 \\
\hline TLR-4-positive Kupffer cells (n) & 0.501 & 0.141 & $<0.001$ & 0.396 & 0.118 & 0.011 \\
\hline BMI z-score & 0.312 & 0.114 & 0.030 & - & - & - \\
\hline Plasma adiponectin & -0.422 & 0.189 & 0.009 & - & - & - \\
\hline $\mathrm{AHI}$ & 0.274 & 0.109 & 0.028 & - & - & - \\
\hline $\mathrm{SaO}_{2}<90 \%(\% \mathrm{TST})$ & 0.270 & 0.134 & 0.031 & - & - & - \\
\hline \multicolumn{7}{|l|}{ Predictors of NAS } \\
\hline $\mathrm{SaO}_{2}<90 \%$ (\%TST) & 0.512 & 0.102 & $<0.001$ & 0.343 & 0.102 & 0.011 \\
\hline Plasma LPS & 0.632 & 0.121 & $<0.001$ & 0.429 & 0.076 & 0.001 \\
\hline TLR-4-positive hepatocytes (\%) & 0.551 & 0.169 & $<0.001$ & 0.352 & 0.089 & 0.009 \\
\hline TLR-4-positive Kupffer cells (n) & 0.579 & 0.133 & $<0.001$ & 0.379 & 0.092 & 0.004 \\
\hline Plasma adiponectin & -0.422 & 0.148 & 0.020 & - & - & - \\
\hline $\mathrm{AHI}$ & 0.311 & 0.192 & 0.030 & - & - & - \\
\hline Adiponectin-positive hepatocytes (\%) & -0.410 & 0.197 & 0.010 & - & - & - \\
\hline Hepatocyte apoptotic index & 0.394 & 0.136 & 0.029 & - & - & - \\
\hline \multicolumn{7}{|l|}{ Predictors of fibrosis stage } \\
\hline $\mathrm{SaO}_{2}<90 \%$ (\%TST) & 0.651 & 0.102 & $<0.001$ & 0.358 & 0.123 & 0.004 \\
\hline Plasma LPS & 0.602 & 0.118 & $<0.001$ & 0.319 & 0.114 & 0.007 \\
\hline TLR-4-positive HSC (n) & 0.528 & 0.162 & $<0.001$ & 0.299 & 0.091 & 0.009 \\
\hline BMI $z$-score & 0.412 & 0.173 & 0.021 & - & - & - \\
\hline $\mathrm{AHI}$ & 0.295 & 0.111 & 0.034 & - & - & - \\
\hline TLR-4-positive Kupffer cells (n) & 0.381 & 0.112 & 0.029 & - & - & - \\
\hline $\mathrm{HSC}(\mathrm{n})$ & 0.421 & 0.113 & 0.019 & - & - & - \\
\hline Adiponectin-positive HPCs (n) & -0.422 & 0.152 & 0.020 & - & - & - \\
\hline
\end{tabular}

Steatosis score, hepatocyte ballooning score, lobular inflammatory score, NAS and fibrosis stage were entered as continuous variables into a multiple linear regression model. $\mathrm{AHI}$, apnoea/hypopnoea index; BMI, body mass index; $\mathrm{ETpCO}_{2}$, mean end-tidal $\mathrm{pCO}_{2} ; \mathrm{HPC}$, hepatic progenitor cell; HSC, hepatic stellate cell; L/M ratio, lactulose/mannitol ratio; LPS, lipopolysaccharide; NAFLD, non-alcoholic fatty liver disease; NAS, NAFLD activity score; ODI, oxygen desaturation index; $\mathrm{SaO}_{2}<90 \%$, total duration of haemoglobin desaturation, expressed as \%TST; TLR-4, toll-like receptor-4.

\section{OSAS is associated with increased intestinal permeability and endotoxemia}

Compared with non-OSAS patients, OSAS was associated with lower plasma adiponectin levels and with increased intestinal permeability and plasma LPS (table 3). Except that for plasma adiponectin statistically significant differences between the above-mentioned parameters were also observed between mild and severe OSAS (table 3).

\section{OSAS and histological, IF and IHC parameters}

Compared with non-OSAS patients, OSAS was associated with a greater prevalence of NASH, and of portal and significant fibrosis, an increased expression of TLR-4 by hepatocytes, Kupffer cells and HSCs, with increased hepatocyte apoptosis and cellcycle arrest indices (table 4). OSAS was also associated with an expansion of HPC compartment and with a reduced adiponectin expression by hepatocytes and HPCs (table 4).

Statistically significant differences between the abovementioned parameters were also observed between mild and severe OSAS (table 4).

\section{Impact of OSAS on liver disease and IHC/IF parameters in} non-obese children

OSAS and obesity often coexist, and obesity has been linked to impaired intestinal barrier function and increased endotoxemia.? We therefore separately assessed the impact of OSAS on gut- 
liver axis and liver IHC/IF parameters in obese and non-obese children with NAFLD (see online supplementary tables S2 and S3): even when restricting the analysis to non-obese participants $(\mathrm{n}=20)$, OSAS was associated with increased intestinal permeability and endotoxemia, increased TLR-4 expression by mature liver cells and expansion of HPCs with reduced adiponectin expression.

To further evaluate the additive effect of BMI and OSAS on endotoxemia, we plotted quartiles of BMI $z$-score and presence/ absence of OSAS: at each BMI quartile, the presence of OSAS was associated with significantly higher plasma LPS levels (figure 1).

\section{Correlative analysis: predictors of liver histology}

The results of univariable and multivariable analyses are reported in tables 5 and 6 .

On multivariable logistic regression analysis, the presence of NASH was predicted by plasma LPS, by $\mathrm{SaO}_{2}<90 \%$ (\%TST), by $\%$ hepatocytes expressing TLR-4 and by the number of Kupffer cells expressing TLR-4 (table 5). The presence of portal fibrosis was predicted by plasma LPS, by the number of HSCs expressing TLR-4 (TLR-4-positive HSCs) and (inversely) by the number of HPCs expressing adiponectin (adiponectin-positive HPCs). The presence of significant (stage $\mathrm{F} \geq 2$ ) fibrosis was predicted by plasma LPS, by $\mathrm{SaO}_{2}<90 \%$ (\%TST) and by the number of HSCs expressing TLR-4 (table 5).

On multivariable linear regression analysis, $\mathrm{SaO}_{2}<90 \%$ (\%TST), plasma LPS and TLR-4 expression by hepatocytes, Kupffer cells and HSCs variably predicted single histological features of NASH, NAS score and fibrosis stage (table 6).

\section{Correlative analysis: predictors of parameters related to gut-liver axis and of hepatic IHC/IF parameters}

The results of univariable and multivariable analyses are reported in tables 7 and 8 .

On multiple linear regression analysis, $\mathrm{SaO}_{2}<90 \%$ (\%TST) independently predicted intestinal permeability (expressed as L/

Table 7 Univariable (left column) and multivariable (right column) linear regression analysis of the predictors of intestinal permeability (L/M ratio) and endotoxemia in the whole NAFLD population $(n=80)$

\begin{tabular}{|c|c|c|c|c|}
\hline \multirow[b]{2}{*}{ Parameter } & \multicolumn{2}{|l|}{ Univariable } & \multicolumn{2}{|l|}{ Multivariable } \\
\hline & $\boldsymbol{\beta}(\mathrm{SE}(\boldsymbol{\beta}))$ & $\mathrm{p}$ Value & $\beta($ SE $(\beta))$ & $\mathrm{p}$ Value \\
\hline \multicolumn{5}{|l|}{ L/M ratio } \\
\hline $\mathrm{SaO}_{2}<90 \%$ (\%TST) & $0.410(0.111)$ & 0.002 & $0.396(0.180)$ & 0.026 \\
\hline $\mathrm{ETpCO}_{2}(\mathrm{~mm} \mathrm{Hg})$ & $0.208(0.131)$ & 0.031 & - & - \\
\hline Systolic BP (mm Hg) & $0.196(0.171)$ & 0.038 & - & - \\
\hline Plasma LPS (EU/mL) & $0.269(0.211)$ & 0.029 & - & - \\
\hline AHI (events/h) & $0.297(0.124)$ & 0.023 & - & - \\
\hline \multicolumn{5}{|l|}{ Plasma LPS } \\
\hline $\mathrm{SaO}_{2}<90 \%$ (\%TST) & $0.692(0.107)$ & $<0.001$ & $0.358(0.107)$ & 0.008 \\
\hline BMI $z$-score & $0.359(0.101)$ & 0.003 & $0.266(0.108$ & 0.019 \\
\hline L/M ratio & $0.418(0.119)$ & 0.001 & $0.366(0.123)$ & 0.006 \\
\hline $\begin{array}{l}\text { Plasma adiponectin } \\
\text { (ng/mL) }\end{array}$ & $-0.356(0.134)$ & 0.009 & - & - \\
\hline AHI (events/h) & $0.268(0.189)$ & 0.027 & - & - \\
\hline Nadir $\mathrm{SaO}_{2}(\% \mathrm{TST})$ & $-0.219(0.121)$ & 0.032 & - & - \\
\hline
\end{tabular}

Only statistically significant associations are shown. Skewed variables were log-transformed.

$\mathrm{AHI}$, apnoea/hypopnoea index; $\mathrm{BMI}$, body mass index; $\mathrm{BP}$, blood pressure; $\mathrm{ETpCO}_{2}$, mean end-tidal $\mathrm{pCO}_{2} ; \mathrm{L} / \mathrm{M}$ ratio, lactulose/mannitol ratio; LPS, lipopolysaccharide; NAFLD, non-alcoholic fatty liver disease; $\mathrm{SaO}_{2}<90 \%$, total duration of haemoglobin desaturation, expressed as \%TST
M ratio), plasma LPS, hepatocyte cell-cycle arrest and apoptotic indices, TLR-4 expression by hepatocytes, Kupffer cells and HSCs, the number of HPCs and (inversely) the number of HPCs expressing adiponectin (tables 7 and 8 and see online supplementary figure S2A-E).

Apoptosis and cell-cycle arrest were assessed by counting the number of hepatocytes that stained strongly positive for cleaved caspase-3 (at the cytoplasm level) and $\mathrm{p} 21^{\text {waf1 }}$ (at the nuclear level). The apoptotic and $\mathrm{p} 21$ indices were calculated by dividing the average number of positive cells by the average number of hepatocytes and expressing the quotient as a percentage for each section.

\section{DISCUSSION}

Novel findings of this study are the following:

1. In paediatric NAFLD, OSAS is characterised by an altered gut-liver axis, represented by an increased intestinal permeability and endotoxemia and by upregulated endotoxin receptor TLR-4 expression by hepatocytes, Kupffer cells and HSCs.

2. OSAS is also characterised by an expanded adiponectindefective HPC pool in the liver.

3. The duration of nocturnal hypoxaemia independently predicts the above-mentioned alterations in the gut-liver axis and in HPC pool.

OSAS has been recently connected to the presence and severity of paediatric NAFLD, independently of whole-body/abdominal obesity, MS and insulin resistance. ${ }^{8} 9$ Unravelling mechanisms mediating OSAS-associated liver injury in NAFLD would have major research and clinical implications, as current therapeutic approaches to paediatric OSAS yield disappointing results: severe OSAS persists in as many as $50 \%$ of children after adeno-tonsillectomy, especially in the presence of obesity and severe OSAS; ${ }^{23}$ CPAP therapy improves polysomnographic parameters and surrogate markers of NAFLD, ${ }^{28}{ }^{29}$ but its feasibility and patient adherence in children remain an issue. ${ }^{10}$ Therefore, it is important to investigate the novel alternative or complementary strategies for the treatment of paediatric OSAS.

An increased LPS-TLR-4 axis activation has been recently implicated in liver injury in NAFLD, ${ }^{12}$ but the mechanisms modulating the LPS-TLR-4 axis activation are unclear. We found that OSAS-related features, and specifically the duration of nocturnal hypoxaemia, were independently associated with increased plasma LPS and TLR-4 expression by hepatocytes, Kupffer cells and HSCs, key features of NASH and fibrosis in our patients (tables 5 and 6).

An increased hypoxia-induced intestinal permeability could account, at least in part, for the increased endotoxemia observed in our population (table 6, see online supplementary figure S2A and $\mathrm{B}$ ): besides facing the anoxic gut lumen, the intestinal mucosa physiologically experiences profound daily fluctuations of perfusion and oxygenation, with the nadir occurring during fasting, a condition that may synergize with nocturnal hypoxaemia to exacerbate gut injury and break intestinal barrier in OSAS; ${ }^{30} 31$ alternatively. hypoxia may shape gut microbiota composition towards LPS-producing bacterial strains.

In our children, OSAS was also associated with increased TLR-4 expression by hepatocytes, Kupffer cells and HSCs, thereby enhancing hepatic susceptibility to circulating LPS (table 5).

Consistent with our findings, growing experimental evidence supports a role for intermittent hypoxia in TLR-4-induced inflammation in different disease models, including myocardial remodelling $^{32}$ and lung and kidney ischaemia-reperfusion 
Table 8 Univariable (left panel) and multivariable (right panel) linear regression analysis of the predictors of different hepatic immunohistochemical and immunofluorescence parameters in the whole NAFLD population $(n=80)$

\begin{tabular}{|c|c|c|c|c|}
\hline \multirow[b]{2}{*}{ Parameter } & \multicolumn{2}{|l|}{ Univariable } & \multicolumn{2}{|l|}{ Multivariable } \\
\hline & $\boldsymbol{\beta}(\mathrm{SE}(\boldsymbol{\beta}))$ & $\mathrm{p}$ Value & $\boldsymbol{\beta}(\mathrm{SE}(\boldsymbol{\beta}))$ & p Value \\
\hline \multicolumn{5}{|l|}{ Hepatocyte cell-cycle arrest index } \\
\hline $\mathrm{SaO}_{2}<90 \%$ (\%TST) & $0.442(0.131)$ & 0.002 & $0.442(0.131)$ & 0.002 \\
\hline Plasma LPS (EU/mL) & $0.389(0.160)$ & 0.002 & - & - \\
\hline HPCs (n) & $0.221(0.112)$ & 0.039 & - & - \\
\hline TLR-4-positive hepatocytes (\%) & $0.228(0.139)$ & 0.028 & - & - \\
\hline \multicolumn{5}{|l|}{ Hepatocyte apoptotic index } \\
\hline $\mathrm{SaO}_{2}<90 \%$ (\%TST) & $0.423(0.101)$ & $<0.001$ & $0.397(0.134)$ & 0.010 \\
\hline Plasma CK18 fragments (U/L) & $0.378(0.113)$ & 0.001 & - & - \\
\hline Plasma LPS (EU/mL) & $0.351(0.109)$ & 0.010 & - & - \\
\hline HPCs (n) & $0.271(0.103)$ & 0.039 & - & - \\
\hline TLR-4-positive hepatocytes (\%) & $0.220(0.127)$ & 0.031 & - & - \\
\hline \multicolumn{5}{|l|}{ TLR-4-positive hepatocytes (\%) } \\
\hline $\mathrm{SaO}_{2}<90 \%$ (\%TST) & $0.695(0.110)$ & $<0.001$ & $0.339(0.112)$ & 0.002 \\
\hline Plasma LPS(EU/mL) & $0.281(0.112)$ & 0.034 & - & - \\
\hline Hepatocyte apoptotic index & $0.304(0.118)$ & 0.015 & - & - \\
\hline Adiponectin-positive hepatocytes (\%) & $-0.290(0.103)$ & 0.030 & - & - \\
\hline $\mathrm{ETpCO}_{2}(\mathrm{~mm} \mathrm{Hg})$ & $-0.221(0.115)$ & 0.029 & - & - \\
\hline TLR-4-positive Kupffer cells (n) & $0.371(0.134)$ & 0.002 & - & - \\
\hline \multicolumn{5}{|l|}{ TLR-4-positive hepatic Kupffer cells (n) } \\
\hline $\mathrm{SaO}_{2}<90 \%(\% \mathrm{TST})$ & $0.655(0.135)$ & $<0.001$ & $0.357(0.135)$ & 0.006 \\
\hline BMI z-score & $0.238(0.104)$ & 0.026 & - & - \\
\hline Plasma LPS(EU/mL) & $0.322(0.128)$ & 0.012 & - & - \\
\hline AHI (events/h) & $0.218(0.101)$ & 0.030 & - & - \\
\hline Nadir $\mathrm{SaO}_{2}(\%)$ & $0.239(0.075)$ & 0.025 & - & - \\
\hline Hepatic TLR-4-positive hepatocytes (\%)* & $0.296(0.128)$ & 0.029 & - & - \\
\hline TLR-4-positive HSCs (n) & $0.358(0.111)$ & 0.009 & - & - \\
\hline Hepatic Kupffer cells (n) & $0.293(0.112)$ & 0.029 & - & - \\
\hline \multicolumn{5}{|l|}{ TLR-4-positive HSCs (n) } \\
\hline $\mathrm{SaO}_{2}<90 \%(\% \mathrm{TST})$ & $0.675(0.137)$ & $<0.001$ & $0.445(0.137)$ & 0.002 \\
\hline BMI z-score & $0.278(0.101)$ & 0.021 & - & - \\
\hline Plasma hyaluronic acid (ng/mL) & $0.348(0.102)$ & 0.010 & - & - \\
\hline Plasma LPS (EU/mL) & $0.399(0.114)$ & 0.008 & - & - \\
\hline AHI (events/h) & $0.245(0.103)$ & 0.021 & - & - \\
\hline Hepatic TLR-4-positive Kupffer cells (n)* & $0.239(0.121)$ & 0.029 & - & - \\
\hline $\operatorname{HSCs}(n)^{*}$ & $0.387(0.103)$ & 0.007 & - & - \\
\hline HPCs (n) & $0.298(0.133)$ & 0.018 & - & - \\
\hline \multicolumn{5}{|l|}{ HPCs (n) } \\
\hline $\mathrm{SaO}_{2}<90 \%(\% \mathrm{TST})$ & $0.650(0.112)$ & $<0.001$ & $0.471(0.191)$ & 0.001 \\
\hline Plasma LPS(EU/mL) & $0.291(0.131)$ & 0.021 & - & - \\
\hline AHI (events/h) & $0.279(0.116)$ & 0.034 & - & - \\
\hline Hepatocyte cell-cycle arrest index (\%) & $0.346(0.153)$ & 0.010 & - & - \\
\hline Hepatocyte apoptotic index (\%) & $0.339(0.123)$ & 0.011 & - & - \\
\hline TLR-4-positive hepatic stellate cells (n) & $0.396(0.118)$ & 0.018 & - & - \\
\hline \multicolumn{5}{|l|}{ Adiponectin-positive HPCs (n) } \\
\hline $\mathrm{SaO}_{2}<90 \%$ (\%TST) & $-0.698(0.119)$ & $<0.001$ & $-0.532(0.141)$ & $<0.001$ \\
\hline Plasma LPS (EU/mL) & $0.319(0.118)$ & 0.013 & - & - \\
\hline AHI (events/h) & $0.272(0.114)$ & 0.034 & - & - \\
\hline Hepatocyte cell-cycle arrest index (\%) & $-0.345(0.125)$ & 0.019 & - & - \\
\hline Hepatocyte apoptotic index (\%) & $-0.299(0.103)$ & 0.030 & - & - \\
\hline Adiponectin-positive hepatocytes (\%) & $0.436(0.112)$ & 0.001 & - & - \\
\hline
\end{tabular}

injury. $^{33} 34$ Intriguingly, TLR-4 antagonists reversed inflammation in these models 33 and could represent a novel therapeutic target for OSAS-associated liver injury, as well.
Another novel finding of our study was the association of nocturnal hypoxaemia with the expansion of an adiponectindefective HPC pool. The expansion of HPC pool following 
chronic liver injury has been proposed to trigger portal fibrosis, an early step in the fibrogenic process in paediatric NASH, ${ }^{1} 13$ but the mechanisms triggering HPC proliferation and their switch to a pro-fibrogenic phenotype are unclear. Our data indicate nocturnal hypoxaemia as a potential regulator of HPC pool size and adiponectin expression, thereby linking OSAS to the pro-fibrogenic HPC activation. ${ }^{13}$

Notably, these associations were independent of BMI and occurred also in non-obese individuals, suggesting that $\mathrm{CIH}$ is per se associated with altered gut-liver axis integrity and hepatic adiponectin expression independently of obesity in NAFLD (table 6; figure 1, see online supplementary tables 1 and 2).

We found no relationship between the severity of liver histology and of OSAS and insulin resistance indices in our patients with NAFLD, which is at odd with part, but not all, of the literature in adult NAFLD. These results are consistent with previous findings in paediatric NAFLD population with OSAS 891113 and confirms common indices of insulin resistance are more tightly related to the presence of fatty liver rather than to the severity of liver histology or sleep apnoea. ${ }^{89}$ An alternative, not exclusive, explanation could be that adipose tissue insulin resistance may be more tightly related to the severity of liver injury and of OSAS than currently used indices, which predominantly reflect hepatic and muscle insulin resistance. ${ }^{35}$

In conclusion, we showed that the CIH of OSAS is accompanied by gut-liver axis dysregulation. Future studies need to assess the causal role of $\mathrm{CIH}$ in these abnormalities, which cannot be ascertained by the cross-sectional design of our study, and to explore potential therapeutic implications of our findings in OSAS-related liver injury, including gut microbiota manipulation and TLR-4 antagonists.

\section{Author affiliations}

${ }^{1}$ Hepato-Metabolic Disease Unit, "Bambino Gesù" Children's Hospital, IRCCS, Rome, Italy

${ }^{2}$ Liver Research Unit, "Bambino Gesù" Children's Hospital, IRCCS, Rome, Italy ${ }^{3}$ Pneumology Unit-Sleep and NIV Laboratory, "Bambino Gesü" Children's Hospital, IRCCS, Rome, Italy

${ }^{4}$ Department of Movement, Human and Health Sciences, Division of Health Sciences, University of Rome "Foro Italico", Rome, Italy

${ }^{5}$ Confocal Microscopy Unit, "Bambino Gesù" Children's Hospital, IRCCS, Rome, Italy

${ }^{6}$ Pathology Unit, "Bambino Gesù" Children's Hospital, IRCCS, Rome, Italy

${ }^{7}$ Department of Anatomical, Histological, Forensic Medicine and Orthopedics

Sciences, Sapienza University of Rome, Rome, Italy

${ }^{8}$ Pediatric Gastroenterology and Liver Unit, Department of Pediatrics, Sapienza University, Rome, Italy

${ }^{9}$ Gradenigo Hospital C.so Regina Margherita 8, Turin, Italy

Contributors VN: conception and design of the study, analysis and/or interpretation of data; revision of the manuscript and approval of the final version of the manuscript. AA, RC, GC, CDS, VD, RDV, SC and EG: generation, collection, assembly, analysis and/or interpretation of data; revision of the manuscript and approval of the final version of the manuscript. GM: conception and design of the study, analysis and/or interpretation of data; drafting of the manuscript and approval of the final version of the manuscript.

Funding The Italian Ministry of Health (fondi di Ricerca Corrente and 5×1000) toVN and $A A$.

Competing interests None declared.

Ethics approval Ethics Committee of the Bambino Gesù Children's Hospital and Research Institute, Rome, Italy.

Provenance and peer review Not commissioned; externally peer reviewed.

Data sharing statement All data are contained in an online database at Banbin Gesù University Hospital, which is available to anyone after justified request to the main author.

\section{REFERENCES}

1 Della Corte C, Vajro P, Socha P, et al. Pediatric non-alcoholic fatty liver disease: Recent advances. Clin Res Hepatol Gastroenterol 2014 38:419-22.

2 Musso G, Gambino R, Cassader M, et al. Meta-analysis: natural history of non-alcoholic fatty liver disease (NAFLD) and diagnostic accuracy of non-invasive tests for liver disease severity. Ann Med 2011;43:617-49.

3 Schwimmer JB, Behling C, Newbury R, et al. Histopathology of pediatric nonalcoholic fatty liver disease. Hepatology 2005;42:641-9.

4 Nobili V, Alisi A, Grimaldi C, et al. Non-alcoholic fatty liver disease and hepatocellular carcinoma in a 7-year-old obese boy: coincidence or comorbidity? Pediatr Obes 2014;9:e99-102.

5 Chalasani N, Younossi Z, Lavine JE, et al. The diagnosis and management of non-alcoholic fatty liver disease: practice Guideline by the American Association for the Study of Liver Diseases, American College of Gastroenterology, and the American Gastroenterological Association. Hepatology 2012;55:2005-23.

6 Bixler EO, Vgontzas AN, Lin HM, et al. Sleep disordered breathing in children in a general population sample: prevalence and risk factors. Sleep 2009;32:731-6.

7 Nevin MA. Pediatric obesity, metabolic syndrome, and obstructive sleep apnea syndrome. Pediatr Ann 2013;42:205-10.

8 Nobili V, Cutrera R, Liccardo D, et al. Obstructive sleep apnea syndrome affects liver histology and inflammatory cell activation in pediatric nonalcoholic Fatty liver disease, regardless of obesity/insulin resistance. Am J Respir Crit Care Med 2014;189:66-76.

9 Sundaram SS, Sokol RJ, Capocelli KE, et al. Obstructive sleep apnea and hypoxemia are associated with advanced liver histology in pediatric non-alcoholic fatty liver disease. J Pediatr 2014;164:699-706.

10 Marcus CL, Brooks LJ, Draper KA. Diagnosis and management of childhood obstructive sleep apnea syndrome. Pediatrics 2012;130:576-84.

11 Giorgio V, Miele L, Principessa L, et al. Intestinal permeability is increased in children with non-alcoholic fatty liver disease, and correlates with liver disease severity. Dig Liver Dis 2014;46:556-60.

12 Frasinariu $\mathrm{OE}$, Ceccarelli $\mathrm{S}$, Alisi $\mathrm{A}$, et al. Gut-liver axis and fibrosis in nonalcoholic fatty liver disease: an input for novel therapies. Dig Liver Dis 2013;45:543-51.

13 Nobili V, Carpino G, Alisi A, et al. Hepatic progenitor stem cells activation, fibrosis, and adipokines production in paediatric non-alcoholic fatty liver disease. Hepatology 2013:56:2142-53.

14 Rhim T, Lee DY, Lee M. Hypoxia as a target for tissue specific gene therapy. J Control Release 2013;172:484-94.

15 Carra GE, Ibáñez JE, Saravi FD. The effect of acute hypoxia on short-circuit current and epithelial resistivity in biopsies from human colon. Dig Dis Sci 2013;58:2499-506.

16 Novosad VL, Richards JL, Phillips NA, et al. Regional susceptibility to stress-induced intestinal injury in the mouse. Am J Physiol Gastrointest Liver Physiol 2013;305: G418-26.

17 Goggins BJ, Chaney C, Radford-Smith GL, et al. Hypoxia and Integrin-Mediated Epithelial Restitution during Mucosal Inflammation. Front Immunol 2013;4:272.

18 Synnestvedt K, Furuta GT, Comerford KM, et al. Ecto-5'-nucleotidase (CD73) regulation by hypoxia-inducible factor- 1 mediates permeability changes in intestinal epithelia. J Clin Invest 2002;110:993-1002.

19 Kuczmarski RJ, Ogden CL, Grummer-Strawn LM. CDC growth charts: United States. Adv Data 2000;314:1-27.

20 Fernández JR, Redden DT, Pietrobelli A, et al. Waist circumference percentiles in nationally representative samples of African-American, European-American, and Mexican-American children and adolescents. J Pediatr 2004;145:439-44.

21 Boney CM, Verma A, Tucker R, et al. Metabolic syndrome in childhood: association with birth weight, maternal obesity, and gestational diabetes mellitus. Pediatrics 2005;115:e290-6.

22 American Academy of Pediatrics, National Cholesterol Education Program. Report of the expert panel on blood cholesterol levels in children and adolescents. Pediatrics 1992;89:525-84.

23 Costa DJ, Mitchell R. Adenotonsillectomy for obstructive sleep apnea in obese children: a meta-analysis. Otolaryngol Head Neck Surg 2009;140:455-60.

24 National High Blood Pressure Education Program Working Group on High Blood Pressure in Children and Adolescents. The fourth report on the diagnosis, evaluation, and treatment of high blood pressure in children and adolescents. Pediatrics 2004;114(2 Suppl 4th Report):555-76.

25 Rosenbloom AL, Silverstein JH, Amemiya S. ISPAD Clinical Practice Consensus Guidelines 2006-2007. Type 2 diabetes mellitus in the child and adolescent. Pediatr Diabetes 2008:9:512-26.

26 Roskams TA, Theise ND, Balabaud C, et al. Nomenclature of the finer branches of the biliary tree: canals, ductules, and ductular reactions in human livers. Hepatology 2004;39:1739-45.

27 Iber C, Ancoli-Israel S, Chesson AL. The AASM manual for the scoring of sleep and associated events: rules, terminology, and technical specifications. 1st edn. Westchester, IL: American Academy of Sleep Medicine, 2007. 
28 Kheirandish-Gozal L, Sans Capdevila O, Kheirandish E, et al. Elevated serum aminotransferase levels in children at risk for obstructive sleep apnea. Chest 2008;133:92-9.

29 Shpirer I, Copel L, Broide E, et al. Continuous positive airway pressure improves sleep apnea associated fatty liver. Lung 2010;188:301-7.

30 Taylor CT, Colgan SP. Hypoxia and gastrointestinal disease. J Mol Med 2007:85:1295-300.

31 Luo $\mathrm{H}$, Guo P, Zhou Q. Role of TLR4/NF- $\mathrm{KB}$ in damage to intestinal mucosa barrier function and bacterial translocation in rats exposed to hypoxia. PLOS ONE 2012;7: e46291.

32 Yuan X, Deng Y, Guo X, et al. Atorvastatin attenuates myocardial remodeling induced by chronic intermittent hypoxia in rats: Partly involvement of
TLR-4/MYD88 pathway. Biochem Biophys Res Commun 2014; 446:292-7.

33 Zhou Z, Zhu X, Chen J, et al. The interaction between Toll-like receptor 4 signaling pathway and hypoxia-inducible factor $1 \alpha$ in lung ischemia-reperfusion injury. J Surg Res 2014;188:290-7.

34 Li YW, Zhang Y, Zhang L, et al. Protective effect of tea polyphenols on renal ischemia/reperfusion injury via suppressing the activation of TLR4/NF- $\kappa B$ p65 signal pathway. Gene 2014;542:46-51.

35 Musso G, Cassader M, De Michieli F, et al. Nonalcoholic steatohepatitis versus steatosis: adipose tissue insulin resistance and dysfunctional response to fat ingestion predict liver injury and altered glucose and lipoprotein metabolism. Hepatology 2012:56:933-42. 